\title{
Categorização hierárquica: revisão de estudos e possibilidades de intervenção para indivíduos com e sem atraso no desenvolvimento
} Hierarchical Categorization: study review and possibilities of intervention on
individuals with and without delayed development Categorización jerárquica: revisión del estudio y posibilidades de intervención en individuos con y sin retraso en el desarrollo

Bruna Nery Rosa ${ }^{1}$, Carolina Coury Silveira ${ }^{1}$, João Henrique de Almeida ${ }^{2}$

[1] Paradigma - Centro de Ciências e Tecnologia do Comportamento [2] Universidade Estadual de Londrina | Título abreviado: Categorização hierárquica | Endereço para correspondência: Carolina Coury Silveira - Benjamin Franklin, 220, casa 38. Bairro Jardim Jamaica, Londrina PR 86 063-240 I Email: carol_coury@hotmail. com I doi: 10.18761/PAC.2021.v12.RFT.18 
Abstract: The Relational Frame Theory (RFT) explains language and cognition based on the Arbitrarily Applicable Relational Responding (RRAA). One of the behaviors that can be explained from this perspective is hierarchical categorization. This study aimed to analyze the literature productions on hierarchical relational frames, focusing on assessment, teaching, and interventions on hierarchical categorization. Based on PRISMA protocol, searches were performed from the CAPES Journal Portal and included bibliographical references. By searching the Researchgate, publications met the following inclusion criteria: empirical studies related to RFT and relational training in hierarchy frame focused on establishing categorization. A total of 15 publications were included in the review and were classified into two categories: (a) Hierarchical Categorization Training and; (b) Others. The review results demonstrated few studies in relational, hierarchical frames, including populations with ASD. However, it was possible to pay attention to the efficiency of teaching procedures and the importance of promoting research in the area, especially in the population with ASD, to improve teaching curricula.

Keywords: Relational Frame Theory; Hierarchical Frame; Autism (ASD).

Resumen: La teoría del marco relacional (RFT) explica el lenguaje y la cognición basándose en la respuesta relacional aplicable arbitrariamente (RRAA). Uno de los comportamientos que se pueden explicar desde esta perspectiva es la categorización jerárquica. Este estudio tuvo como objetivo analizar las producciones de la literatura sobre marcos relacionales jerárquicos, enfocándose en la evaluación, la enseñanza y las intervenciones sobre la categorización jerárquica. Con base en el protocolo PRISMA, las búsquedas se realizaron desde el Portal de Revistas CAPES e incluyeron referencias bibliográficas. Al buscar en el Researchgate, las publicaciones cumplieron con los siguientes criterios de inclusión: estudios empíricos relacionados con RFT y entrenamiento relacional en el marco de jerarquía enfocado en establecer categorización. Se incluyeron un total de 15 publicaciones en la revisión y se clasificaron en dos categorías: (a) Capacitación en categorización jerárquica y; (b) Otros. Los resultados de la revisión demostraron pocos estudios en marcos jerárquicos relacionales, incluidas poblaciones con TEA. Sin embargo, se pudo prestar atención a la eficiencia de los procedimientos de enseñanza y la importancia de promover la investigación en el área, especialmente en la población con TEA, para mejorar los planes de estudio de la enseñanza.

Palabras clave: Teoría del marcos relacionales; Marco jerárquico; Autismo (TEA). 
Em um contexto teórico amplo, categorização ou classificação podem ser definidas como processos pelo qual elementos podem ser agrupados, reconhecidos, diferenciados e classificados em determinados grupos ou categorias, visando um propósito definido (Laipelt \& Krebs, 2018). Na categorização hierárquica as próprias classes de elementos são categorizadas em organizações ou classes de níveis hierárquicos diferentes, ou seja, em grupos maiores e genéricos que os incluem ou elementos inferiores como elementos ou conjuntos de elementos incluídos nesses grupos (Griffee \& Dougher 2002; Slattery \& Stewart, 2014). Os estudos iniciais em categorização hierárquica foram conduzidos no campo da Psicologia Cognitiva (e.g. Blewitt, 1994) e pesquisas neste campo sugerem que este conjunto de habilidades é adquirido gradualmente, a partir da primeira infância com comportamentos de categorização mais básicos atingindo outros comportamentos de categorização hierárquica mais complexa no início da adolescência (para uma revisão mais aprofundada nesse tema ver (Carneiro, Albuquerque \& Fernandez, 2009; Eimas \& Quinn, 1994 ).

A categorização hierárquica é um comportamento complexo e altamente relevante que comumente é avaliado em testes psicológicos (e.g., SON-R). Segundo Ming et al., (2018), uma maneira de avaliar essa resposta é o teste chamado de teste de inclusão de classe. Neste teste, a criança vê uma série de estímulos pertencentes a uma classe particular que inclui duas subclasses diferentes, com maior quantidade de estímulos de uma das duas subclasses. As crianças são questionadas se há mais membros dos correspondentes a quantidade de itens em uma subclasse ou mais membros de toda a classe. Por exemplo, "Tem mais cachorros ou mais animais?". Este tipo de pergunta permite investigar a resposta de categorização baseado em classes e subclasses.

Como apresentado por McCabe, Seigal, Spence e Wilkinson (1982), participantes entre 3 e 8 anos foram apresentados a uma classe de doces: jujubas, balas e M\&Ms, em sequência. Em seguida o experimentador perguntava à criança se haviam mais M\&Ms ou mais doces. Verificou-se que os participantes de 3 e 4 anos responderam de maneira aleatória, enquanto os participantes de 7 e 8 anos responderam corretamente. Obviamente, devi- do a quantidade de estudos realizados com uma abordagem amplamente descritiva pela Psicologia Cognitiva sobre esse tema, inúmeras variáveis maturacionais relevantes foram identificadas (Murphy, 2002). No entanto, esses estudos frequentemente negligenciam a influência de variáveis ambientais no controle do comportamento (Slattery \& Stewart,2014). Por esta razão, era imprescindível que o tema da categorização hierárquica fosse investigado a partir do escopo da Análise do Comportamento. Desde o início dos anos 2000, esses trabalhos começaram a ser desenvolvidos, embasados especialmente na Teoria das Molduras Relacionais (Relational Frame Theory - RFT). A RFT é uma teoria comportamental contemporânea para explicação da linguagem e cognição humana (Hayes, Barnes-Holmes \& Roche, 2001).

A RFT descreve o comportamento verbal como um comportamento complexo e a partir desse comportamento é possível definir funcionalmente a categorização hierárquica, que pode ser compreendida como relações generalizadas e contextualmente controladas. Em outras palavras a categorização hierárquica pode ser definida com responder relacional arbitrariamente aplicável (RRAA). O controle contextual que dá origem as RRAAs é aprendido a partir de Treinos de Múltiplos Exemplares, com relações arbitrárias e não arbitrárias entre os elementos (Perez, Nico, Kovac, Fidalgo, \& Leonardi, 2013; Ming et al. 2017).

Classicamente as RRAAs apresentam três propriedades definidoras: (a) Implicação Mútua, que se refere à bidirecionalidade da resposta relacional, (b) Implicação Mútua Combinatória, que se refere a uma relação de estímulo derivada em que duas ou mais relações de estímulo combinam-se mutuamente e (c) Transformação de Função de estímulo, que se refere à transformação de funções psicológicas relevantes de um estímulo, de acordo com a relação derivada subjacente em um determinado contexto. Este tipo de comportamento permite que as funções de um evento sejam determinadas indiretamente, graças a sua participação em redes relacionais derivadas (McHugh \& Reed, 2008).

Para a RFT, a categorização hierárquica é um tipo de moldura relacional, conhecida como Moldura Relacional Hierárquica. Ela é estabelecida a partir de dicas contextuais hierárquicas em trei- 
nos de múltiplos exemplares, como por exemplo quando em uma interação com os pais ou responsáveis, a criança gradualmente aprende a dizer que "uma bola está dentro de uma caixa" ou mesmo que "a caixa contém a bola", considerando relações não-arbitrárias. Adicionalmente, outro exemplo desse tipo de aprendizagem de dicas contextuais relacionais de hierarquia ocorre quando a criança aprende que "um gato é um tipo de felino" e que "o conjunto dos felinos inclui gatos, onças e tigres", potencialmente ensinando relações arbitrárias entre estímulos (Hayes et al., 2001). Diferentemente das relações de coordenação ou de equivalência em que a bidirecionalidade entre os estímulos é "simétrica", no caso das relações hierárquicas se "A está contido em B", a implicação mútua permitirá dizer que "B contém A" (Slattery \& Stewart, 2002; Slattery, Stewart \& O’Hora, 2011).

Em um primeiro momento, os estudos comportamentais tiveram um enfoque em produzir uma explicação da categorização hierárquica (Griffee \& Dougher, 2002). Griffee e Dougher investigaram a categorização hierárquica como variável dependente de um procedimento de treino que consistiu em treinamento de discriminação condicional contextualmente controlada, generalização de estímulos e equivalência de estímulos. A aquisição da habilidade de categorização foi observada, utilizando um procedimento que colocou sob controle contextual respostas a diferentes estímulos (triângulos, símbolos sem sentido e cores de fundo de tela) de maneira hierárquica. A característica que tornou essas relações, também, hierárquicas é que existiam alguns elementos mais abrangentes ou gerais "no topo" desta hierarquia e outros mais específicos nos níveis mais baixos. Os autores afirmaram a categorização uma vez que, resumidamente, as propriedades dos níveis superiores foram transformadas para os níveis inferiores e raramente o contrário. No entanto, seus testes utilizaram estímulos fisicamente semelhantes aos estímulos do treino e os desempenhos observados poderiam também ser atribuídos a generalização de estímulos.

Com o intuito de demonstrar esse repertório comportamental, sem que os resultados pudessem ser atribuídos a generalização, Slattery, Stewart e O'Hora (2011) expandiram os procedimentos realizados anteriormente. Nesse segundo estudo, os autores exploraram uma das características da categorização hierárquica mais conhecida nos estudos da Psicologia Cognitiva, sendo ela a contenção de classe transitiva. Para tanto, após a exposição a treino de discriminação condicional e exposição a múltiplos exemplares, os participantes passariam em um teste para responder de acordo com contenção de classe transitiva. Os procedimentos de todos os estudos realizados aqui foram muito semelhantes ao desenvolvido por Griffee e Dougher. Slattery e colaboradores empregaram também diferentes triângulos com diferentes cores de fundo de tela. Os fundos de tela contextualmente permitiam que os participantes respondessem em diferentes níveis hierárquicos diante dos triângulos, e posteriormente diante destes triângulos respondessem a silabas sem sentido considerando o formato do triângulo e a cor do fundo da tela. Foram realizados três experimentos e no terceiro e último experimento descrito pelos autores foi possível observar o pertencimento de classe transitiva para todos os três participantes de maneira independente a características físicas dos estímulos. Isto é, foi uma demonstração clara da explicação da RFT para categorização hierárquica. Estes trabalhos iniciais permitiram um grande avanço na maneira com que a Análise do Comportamento poderia interpretar e intervir neste tipo de repertório.

A precisão que a RFT apresenta em sua definição de categorização hierárquica permite entender funcionalmente como crianças com desenvolvimento típico começam a apresentar esse tipo de resposta em seu repertório comportamental. No entanto, algumas crianças com desenvolvimento atípico podem apresentar dificuldades em aprender tais habilidades apenas por treinos incidentais, isto é, somente com as oportunidades dadas pelo contexto natural. Procedimentos como os relatados anteriormente, vêm demonstrando resultados satisfatórios no treino dessas respostas para crianças com atraso no desenvolvimento (Ming et al., 2018; Zagrabska-Swiatkowska, Mulhern, Ming, Stewart, \& McElwee, 2020).

Dada a relevância desse repertório comportamental e a eficácia observada por intervenções baseadas em RFT para o ensino de categorização hierárquica, o objetivo do presente estudo foi analisar um conjunto posterior das produções de literatura 
sobre molduras de hierarquia a fim de investigar quais as características do estabelecimento desse repertório em relação a sua eficácia e efetividade. Esse conjunto, constituí uma série de estudos desenvolvidos a partir de 2011 que em seus procedimentos incluíam características mais próximas de intervenções ou aplicações ou mesmo a transformação de funções, centrados no repertório de categorização hierárquica. Esse conjunto de estudos apresenta procedimentos mais prontamente adaptáveis para um contexto de aplicação. Dessa maneira, constituíram o foco desse trabalho, estudos que investigaram como a explicação da RFT pode contribuir com a compreensão da categorização hierárquica, suas propriedades ou a transformação de funções a partir desse tipo de controle contextual. A partir da revisão destes estudos com esse caráter, o segundo objetivo foi discutir as possibilidades de intervenção neste tipo de repertório para indivíduos com TEA, vislumbrando o desenvolvimento de novas tecnologias de ensino.

\section{Método}

O Protocolo PRISMA pauta o método dessa pesquisa. Tal método consiste nas seguintes etapas: i) identificação; ii) seleção de artigos; iii) elegibilidade e iv) inclusão. A primeira etapa, identificação, consistiu em buscas bibliográficas na base de dados acadêmica: Portal de Periódicos (CAPES), em que a psicologia foi selecionada como área do conhecimento filtrada. Os termos de pesquisa que determinaram as palavras-chaves inseridas foram: "Teoria das molduras relacionais" AND "Hierarquia"; "Relational Frame Theory" AND "Hierarchical Relations"; "Relational Frame Theory" AND "Frame of Hierarchy"; "Relational Frame Theory" AND "Hierarchy"; "Relational Frame Theory" AND "Hierarchy" AND "Autism"; "Relational Frame Theory" AND "Class Inclusion" e; "Relational Frame Theory" AND "Hierarchical Class". As palavras chaves foram combinadas, nas buscas realizadas, a partir do uso do operador booleano and, seguindo o padrão apresentado na Tabela 1.

Para a etapa de seleção, foi realizada a leitura dos títulos e resumos dos periódicos localizados. Foram selecionados aqueles que se adequaram ao critério de inclusão por serem estudos relacionados à Teoria das Molduras Relacionais (RFT) e estudos com treino relacional na moldura de hierarquia. Já os critérios de exclusão foram estudos e discussões teóricas, revisões de literatura, comentários de estudos anteriores e estudos com treino relacional em outras molduras relacionais que não incluíssem a hierarquia.

As buscas englobaram estudos publicados até março de 2021, e foram realizadas por duas pesquisadoras, de forma independente. Ambas realizaram a busca na base de dados para a seleção dos periódicos encontrados seguindo os critérios de inclusão, denominados assim como "incluídos", os que não atendiam aos critérios de inclusão ou atendiam aos critérios de exclusão foram categorizados como "excluídos", já periódicos encontrados em mais de uma busca foram classificados como "duplicados". Foi utilizado o índice de concordância Kappa para avaliar a concordância entre as duas pesquisadoras,

Tabela 1. Descrição da busca na base de dados

\begin{tabular}{ll}
\hline Base & Descritores (palavras + operadores) \\
\hline & "Teoria das molduras relacionais" AND Hierarquia \\
& "Relational Frame Theory" AND "Hierarchical Relations" \\
& "Relational Frame Theory" AND "Frame of Hierarchy" \\
Catálogo de Teses e Dissertações (CAPES) & "Relational Frame Theory" AND "Hierarchy" \\
& "Relational Frame Theory" AND "Hierarchy" AND "Autism" \\
& "Relational Frame Theory" AND "Class Inclusion" \\
& "Relational Frame Theory" AND "Hierarchical Class" \\
\hline
\end{tabular}


indicando concordância de $\mathrm{k}=1 \mathrm{em}$ uma escala e 0 a 1 , no qual 1 corresponde a concordância total. $\mathrm{Na}$ etapa de elegibilidade, foram excluídos os registros que estavam duplicados.

$\mathrm{Na}$ etapa de inclusão, foram incluídos registros localizados por meio de outras fontes de informação, como indicação de pesquisadores, seção de referências dos artigos recuperados e busca por publicações dos principais autores de molduras relacionais de hierarquia no Researchgate ${ }^{\oplus}$. Os dados do processo de busca bibliográfica constam na Figura 1.

$\mathrm{Na}$ fase de identificação foram encontrados 145 registros. Foram lidos título e resumo de todos os registros e resultaram apenas 24 que atenderem aos critérios de inclusão. Dos 24 registros restantes, 13 eram duplicados, restando 11 cujos textos foram recuperados. Outros artigos foram recuperados por outras fontes de informações (já excluindo os duplicados que haviam sido recuperados na busca bibliográfica): três artigos foram localizados nas sessões de referências dos artigos previamente re-

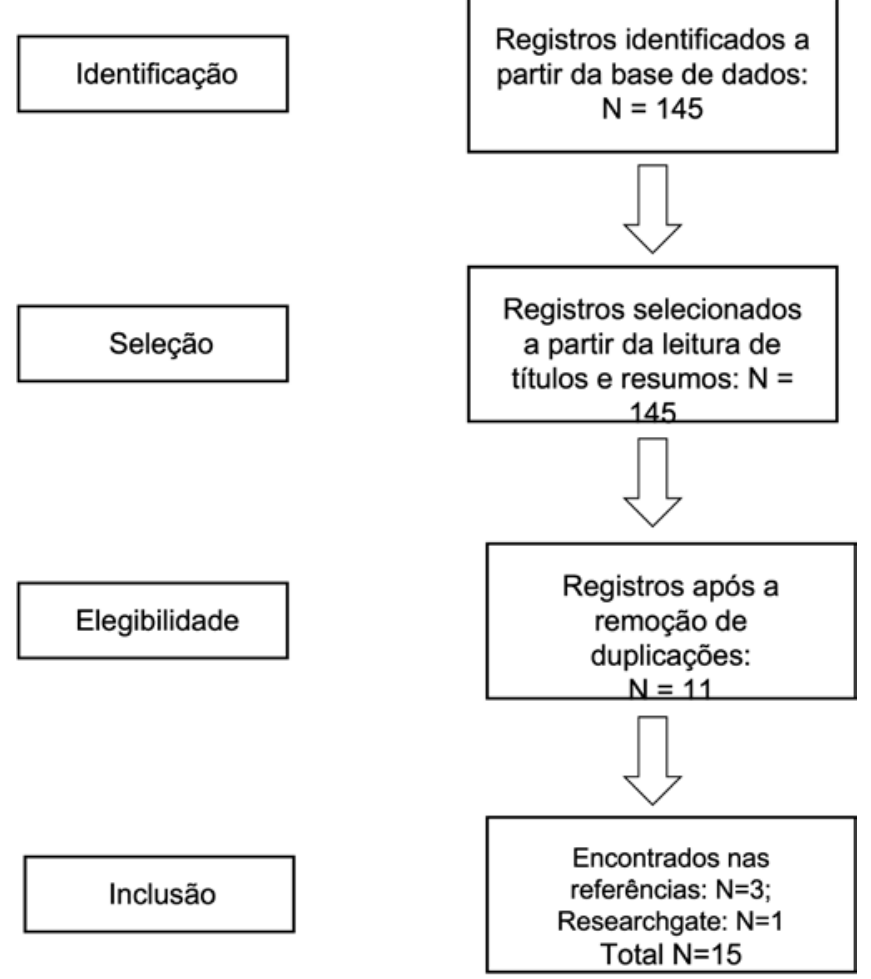

Figura 1. Fluxograma com os resultados encontrados na base de dados cuperados, um artigo foi proveniente da busca pelo Researchgate ${ }^{\circledast}$. Os 15 estudos incluídos nesta revisão sistemática foram lidos na íntegra e as seguintes informações foram extraídas e organizadas em uma tabela do Microsoft Excel ${ }^{\oplus}$ para análise: (a) autores, (b) ano, (c) título, (d) periódico, (e) objetivos, (f) caracterização dos participantes (número, faixa etária, população e escolaridade), (g) delineamento, (h) local, (i) aspectos do procedimento (pré e pós-teste, tipos de treinos e testes e dica contextual), (j) número de sessões, (k) principais resultados, (l) limitações indicadas pelos autores, e (m) verificação se o estudo é favorável à definição da RFT.As características formais coletadas foram analisadas por meio de estatística descritiva, utilizando cálculo de porcentagem para identificar a sua frequência de ocorrência. Foram elas o ano de publicação e o periódico de publicação. As características metodológicas foram analisadas levando em conta (a) o objetivo dos estudos; (b) os participantes dos estudos; (c) o procedimento utilizado; e (d) os resultados alcançados.

\section{Resultados}

A busca de artigos no portal do CAPES resultou inicialmente na identificação de 145 ocorrências sob os filtros "Teoria das molduras relacionais" AND "Hierarquia"; "Relational Frame Theory" AND "Hierarchical Relations"; "Relational Frame Theory" AND "Frame of Hierarchy"; "Relational Frame Theory" AND "Hierarchy"; "Relational Frame Theory" AND "Hierarchy" AND "Autism"; "Relational Frame Theory" AND "Class Inclusion" e; "Relational Frame Theory" AND "Hierarchical Class". A amostra foi reduzida em aproximadamente $92 \%(n=134)$ ao serem aplicados os critérios de exclusão e retirada de duplicações, totalizando assim, em 11 periódicos encontrados no Portal de Periódicos da CAPES. No entanto, foram acrescidos aos resultados mais quatro publicações, sendo três encontradas nas referências dos artigos e mais um manuscrito obtido pelo Researchgate ${ }^{\circledR}$. Obteve-se como resultado, 15 periódicos encontrados, publicados entre os anos de 2011 e 2021, como pode ser observado na Figura 2. Foi possível notar que as publicações são relativamente recen- 


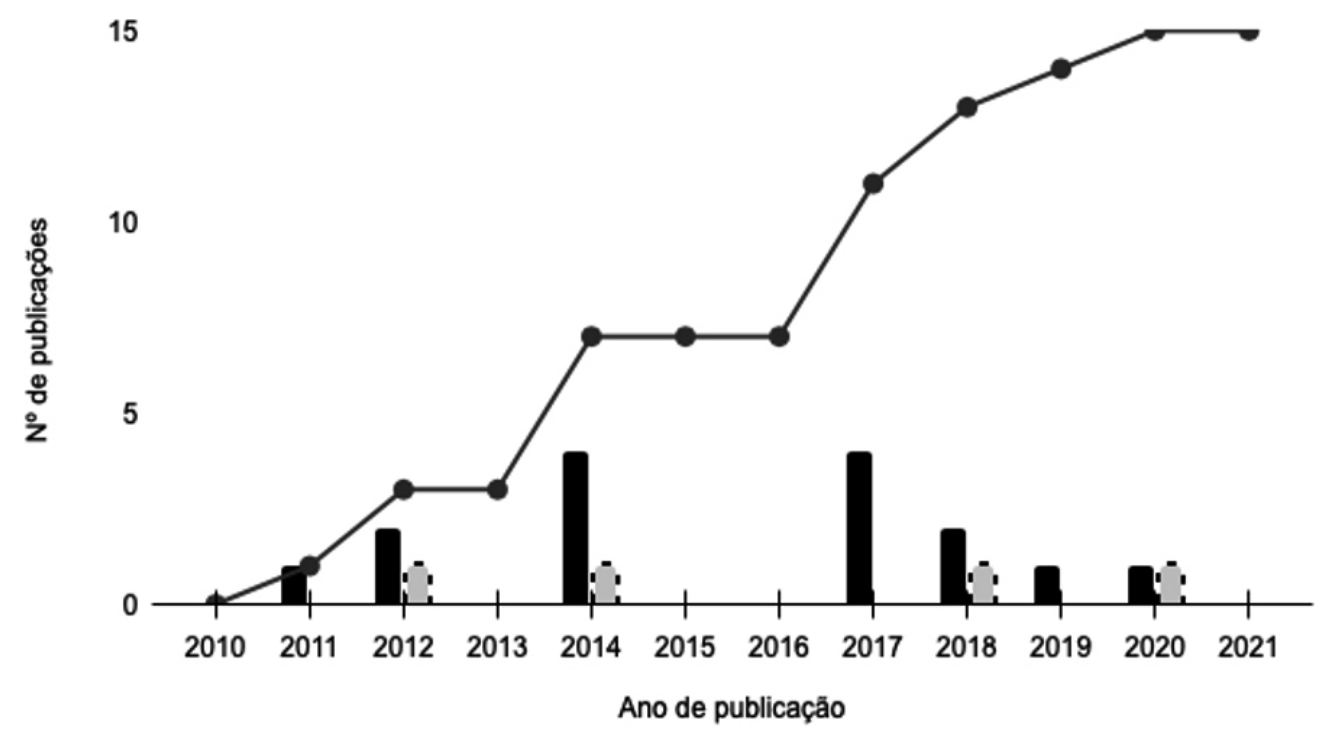

Figura 2. Número de publicações por ano e curva acumulada no período de 2011 a 2021.

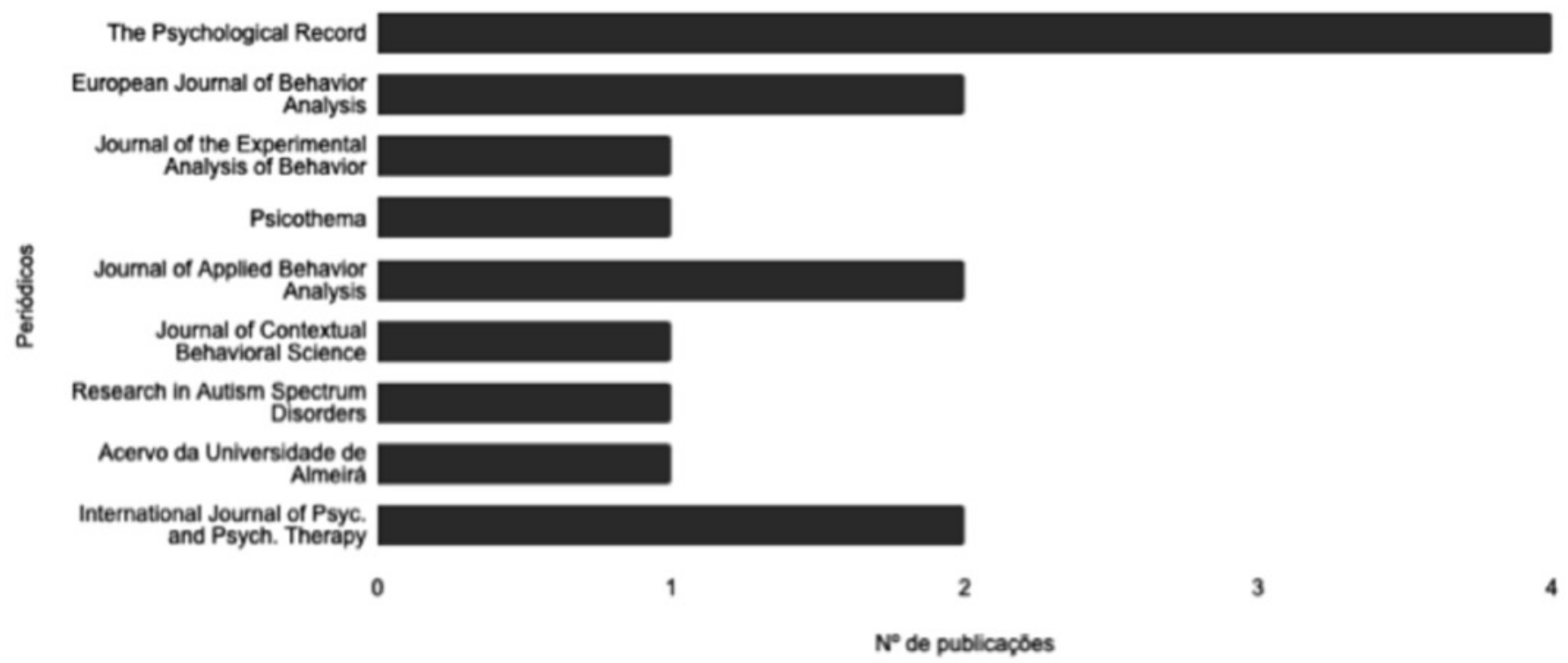

Figura 3. Número de artigos por periódico de publicação 
Tabela 2. Categorização das publicações segundo os objetivos dos estudos.

\begin{tabular}{|c|c|c|c|c|}
\hline Categoria & Autores/Ano & Objetivos & Participantes & Faixa Etária \\
\hline \multirow{10}{*}{$\begin{array}{l}\text { Treinos de } \\
\text { Categoriza. } \\
\text { Hierárquica }\end{array}$} & Gil, et al., (2012) & $\begin{array}{l}\text { Analisar algumas das transformações de funções } \\
\text { que ocorrem em diferentes níveis de categorias } \\
\text { hierárquicas. }\end{array}$ & Adultos & 18 a 33 anos \\
\hline & $\begin{array}{l}\text { Slattery, \& Stewart } \\
(2014)\end{array}$ & $\begin{array}{l}\text { Modelar a categorização hierárquica sob controle } \\
\text { contextual. }\end{array}$ & Adultos & 22 a 27 anos \\
\hline & Gil, et al., (2014) & $\begin{array}{l}\text { Analisar a transformação de função por meio de } \\
\text { relações hierárquicas. }\end{array}$ & Adultos & 17 a 39 anos \\
\hline & Ming, et al., (2017) & $\begin{array}{l}\text { Avaliar e treinar a habilidade responder relacional } \\
\text { hierárquico/ inclusão de classe. }\end{array}$ & $\begin{array}{l}\text { Crianças com } \\
\text { e sem TEA }\end{array}$ & 3 a 19 anos \\
\hline & $\begin{array}{l}\text { Mulhern, et al., } \\
\text { (2017). }\end{array}$ & $\begin{array}{l}\text { Investigar responder relacional em relação à cate- } \\
\text { gorização hierárquica em crianças }\end{array}$ & Crianças & 3 a 8 anos \\
\hline & $\begin{array}{l}\text { Mulhern \& Stewart } \\
(2018)\end{array}$ & $\begin{array}{l}\text { Avaliar e treinar o enquadramento relacional de } \\
\text { categorização em crianças com desenvolvimento tí- } \\
\text { pico e avaliar o impacto de treinamento em medidas } \\
\text { relevantes de linguagem e categorização }\end{array}$ & Crianças & 5 a 6 anos \\
\hline & $\begin{array}{l}\text { Stewart et al., } \\
(2018)\end{array}$ & $\begin{array}{l}\text { Investigar a resposta hierárquica do tipo de análise } \\
\text { ("parte-todo") }\end{array}$ & Adultos & 21 a 29 anos \\
\hline & $\begin{array}{l}\text { Zagrabska } \\
\text { Swiatkowska, et } \\
\text { al.,(2020) }\end{array}$ & $\begin{array}{l}\text { Extensão do estudo de Ming et. al (2018). Avaliar e } \\
\text { treinar a habilidade de responder relacional hierár- } \\
\text { quico/ inclusão de classe }\end{array}$ & $\begin{array}{l}\text { Adultos com } \\
\text { TEA }\end{array}$ & 23 a 26 anos \\
\hline & $\begin{array}{l}\text { Persicke et al., } \\
\text { (2012) }\end{array}$ & $\begin{array}{l}\text { Avaliar se a eficácia do treino de múltiplos exempla- } \\
\text { res para ensinar crianças com autismo de maneira } \\
\text { conjunta com uso de metáfora. }\end{array}$ & $\begin{array}{l}\text { Crianças com } \\
\text { TEA }\end{array}$ & 5 a 6 anos \\
\hline & $\begin{array}{l}\text { Brooks Newsome } \\
\text { et al., (2014) }\end{array}$ & $\begin{array}{l}\text { Avaliaram os efeitos da exposição a treino de } \\
\text { múltiplos exemplares para compreensão de leitura: } \\
\text { a partir do treino relacional de hierarquia, coordena- } \\
\text { ção e diferença }\end{array}$ & $\begin{array}{l}\text { Crianças com } \\
\text { TEA }\end{array}$ & 9 a 12 anos \\
\hline \multirow{4}{*}{ Outros } & $\begin{array}{l}\text { Gil-Luciano et al., } \\
\text { (2017) }\end{array}$ & $\begin{array}{l}\text { Analisar o efeito de dois protocolos de desfusão } \\
\text { definidos por RFT na promoção da flexibilidade } \\
\text { psicológica, alterando as funções discriminativas de } \\
\text { esquivar-se de eventos privados aversivos. }\end{array}$ & Adultos típicos & 21 a 46 anos \\
\hline & $\begin{array}{l}\text { López-López } \\
\text { (2017) }\end{array}$ & $\begin{array}{l}\text { Analisar o efeito de diferentes tipos de enqua- } \\
\text { dramento do próprio comportamento, como nas } \\
\text { interações de desfusão, no desempenho em várias } \\
\text { tarefas experimentais }\end{array}$ & Adultos típicos & 18 a 33 anos \\
\hline & $\begin{array}{l}\text { Luciano et al., } \\
\text { (2019) }\end{array}$ & $\begin{array}{l}\text { Testar a hipótese de que gatilhos de pensamentos } \\
\text { negativos repetitivos são geralmente relacionados } \\
\text { hierarquicamente }\end{array}$ & Adultos típicos & 18 a 32 anos \\
\hline & $\begin{array}{l}\text { Foody et al., } \\
(2014)\end{array}$ & $\begin{array}{l}\text { Investigar a distinção e as relações hierárquicas } \\
\text { quando direcionadas especificamente para um } \\
\text { exercício de "eu como contexto". }\end{array}$ & Adultos típicos & 17 a 41 anos \\
\hline
\end{tabular}

tes, uma vez que o primeiro livro apresentando a proposta da RFT foi publicado no ano de 2001 e a proposta original data dos anos 80 . Desses textos publicados, apenas um é em língua espanhola e os outros 14 foram publicados em língua inglesa. $\mathrm{O}$ trabalho publicado em língua espanhola, trata-se de uma tese de doutorado que foi comentado em uma seção posterior neste trabalho, mas que não confi- gurou parte direta da análise. Dessa forma o escopo dessa revisão considerou 14 artigos deste conjunto de textos com aplicações passíveis de adaptação a contextos educacionais ou clínicos e com foco em intervir no repertório de categorização hierárquica.

Ao longo dos 10 anos a partir da primeira publicação há uma média de 1,5 artigos publicados anualmente. No entanto, a Figura 2 descreve inter- 
valos de publicações, como por exemplo nos anos de 2013 e 2016, em que nenhuma publicação foi encontrada. Por outro lado, nos últimos quatro anos, a partir de 2017, pesquisas em molduras relacionais de hierarquia estão sendo publicadas em uma média de duas publicações ao ano, o que aponta uma tendência ascendente na curva acumulada. Dentre todas as publicações encontradas, apenas $26,6 \%$ $(\mathrm{n}=4)$ possuíam como participantes pessoas com desenvolvimento atípico, sendo uma com participantes de desenvolvimento típico e autismo e três constando apenas participantes de desenvolvimento atípico, como Síndrome de Down e Autismo. As publicações empíricas sobre molduras relacionais de hierarquia estão presentes em nove revistas científicas, como demonstrado na Figura 3. O periódico The Psychological Record ( $\mathrm{n}=4 ; 25 \%)$ desponta nas publicações de materiais empíricos na área. Porém, materiais empíricos que possuam como participantes pessoas com desenvolvimento atípico estão presentes apenas em três revistas, sendo elas: Journal of Applied Behavior Analysis, com duas publicações, representando $50 \%$ das publicações de periódicos com público com desenvolvimento atípico, seguido do European Journal of Behavior e Research in Autism Spectrum Disorders, cada um com uma publicação.

Em relação às características metodológicas, as publicações foram divididas em duas categorias: Categoria 1 - Procedimentos investigando categorização hierárquica e Categoria 2 - Outros. As duas categorias foram analisadas, a partir dos seguintes tópicos (a) objetivo; (b) autores; (c) tipo de participantes; (d) faixa etária dos participantes. Esses dados foram apresentados na Tabela 1. A categoria "Outros", não foi apresentada com detalhes em uma seção posterior por compreenderem intervenções utilizando relações ou dicas contextuais de hierarquia de forma muito diversa do escopo dos objetivos desse estudo, como avaliar a efetividade de treinos de metáforas, verificar compreensão de leitura e especialmente no contexto de intervenções terapêuticas, em sua maioria em adultos com desenvolvimento típico.

\section{Procedimentos Investigando Categorização Hierárquica}

Esses procedimentos foram apresentados em oito estudos, dos quatorze totais encontrados: Gil, Luciano, Ruíz e Valdivia-Salas (2012; 2014); Slattery e Stewart (2014); Ming, Mulhern, Stewart, Moran e Bynum. (2017); Mulhern, Stewart e McElwee (2017; 2018); Stewart, Slattery, Chambers e Dymond. (2018); e Zagrabska-Swiatkowska,et al. (2020). Nesses estudos, os participantes foram expostos a procedimentos que ensinaram relações não-arbitrárias e/ou arbitrárias de hierarquia, e testaram as relações derivadas em molduras relacionais hierárquicas.

Em relação aos participantes, a faixa etária variou de crianças pré-escolares com 3 anos de idade a adultos com 39 anos. Nesses estudos, apenas dois contaram com participantes com Transtorno do Espectro Autista (TEA) (Ming, Mulhern, Stewart, Moran \& Bynum, 2018; e Zagrabska-Swiatkowska, et al., 2020), os demais contaram com participantes com desenvolvimento típico. Um detalhe importante a ser descrito é a variação de faixa etária do estudo Ming et. al (2018), no qual consta participantes com faixa etária de 3 a 19 anos de idade. Neste estudo estão presentes crianças de desenvolvimento típico entre 3 e 4 anos de idade e crianças com TEA de 8, 9 e 19 anos, que foram avaliadas pelo Peabody Picture Vocabulary Test, Fourth Edition ${ }^{\circledR}$ (PPVT-4; Dunn \& Dunn, 2007) e apresentaram repertório de linguagem equivalentes à de crianças com 5, 6 e 7 anos, respectivamente.

As características analisadas dos procedimentos realizados em cada um desses estudos da seção de Treino das Molduras Relacionais de Hierarquia foram: número de participantes; formato do treino; avaliação do repertório de entrada; dica contextual utilizada; ocorrência ou não treinos de relações não-arbitrárias e arbitrárias; se foram realizados testes das propriedades definidoras de Implicação Mútua (IM), Implicação Mútua Combinatória (IC) e Transformação de Função (TOF); e por fim, proporção da efetividade do treino. Estas características estão apresentadas na Tabela 3 .

A tese de doutorado de Gil (2011) não foi incluída na contagem dos oito estudos e a justificativa desta escolha será apresentada a seguir. Tal tese contém os primeiros experimentos que exploram mol- 
Tabela 3. Dados do procedimento dos estudos de treino de hierarquia

\begin{tabular}{|c|c|c|c|c|c|c|c|c|c|c|}
\hline \multirow[t]{2}{*}{ Autores/Ano } & \multicolumn{10}{|c|}{ Características do Procedimento } \\
\hline & $\begin{array}{l}\text { Avaliação do } \\
\text { Repertório } \\
\text { de Entrada }\end{array}$ & Dicas Contextuais & $\begin{array}{l}\text { Treinos de } \\
\text { Relações } \\
\text { Arbitrárias }\end{array}$ & $\begin{array}{l}\text { Fase } \\
\text { Arbitrária }\end{array}$ & IM & IMC & TOF & $\mathrm{N}$ & $\begin{array}{l}\text { Formato do } \\
\text { Treino }\end{array}$ & Efetividade \\
\hline Gil et al., (2012) & Não & $\begin{array}{l}\text { Inclui, pertence } a \text {, } \\
\text { mesmo e diferente }\end{array}$ & Imagens & Sim & Não & Não & Sim & 10 & Computador & Sim \\
\hline $\begin{array}{l}\text { Slattery \& } \\
\text { Stewart, (2014) }\end{array}$ & História & Membro de, inclui & Imagens & Sim & Sim & Sim & Sim & 10 & Computador & Sim \\
\hline Gil et al., (2014) & História & $\begin{array}{l}\text { Inclui, pertence a, } \\
\text { mesmo e diferente }\end{array}$ & Imagens & Sim & Sim & Sim & Sim & 6 & Computador & Sim \\
\hline $\begin{array}{l}\text { Ming, et al., } \\
(2018)\end{array}$ & $\begin{array}{l}\text { Avaliação } \\
\text { PPVT-4 e } \\
\text { Linha de } \\
\text { Base }\end{array}$ & Mais e Menos & $\begin{array}{l}\text { Estímulos } \\
\text { físicos }\end{array}$ & Sim & - & - & - & 6 & Mesa & Sim \\
\hline $\begin{array}{l}\text { Mulhern, Stewart } \\
\text { \& McElwee } \\
\text { (2017) }\end{array}$ & $\begin{array}{l}\text { PPVT-4, } \\
\text { CCT, SB5 }\end{array}$ & Está dentro e contém & $\begin{array}{l}\text { Estímulos } \\
\text { físicos e } \\
\text { imagens }\end{array}$ & Sim & Sim & Sim & Sim & 50 & Mesa/Comp. & - \\
\hline $\begin{array}{l}\text { Mulhern, Stewart } \\
\text { \& McElwee } \\
\text { (2018) }\end{array}$ & $\begin{array}{l}\text { PPVT-4, } \\
\text { CCT e } \\
\text { inclusão de } \\
\text { classe (IC). }\end{array}$ & Está dentro e contém & $\begin{array}{l}\text { Estímulos } \\
\text { físicos }\end{array}$ & Sim & Sim & Sim & Não & 12 & Mesa/Comp. & Sim \\
\hline $\begin{array}{l}\text { Stewart et al., } \\
(2018)\end{array}$ & Sim & "Parte de" e "inclui" & Imagens & Sim & Sim & Sim & Sim & 10 & Computador & Sim \\
\hline $\begin{array}{l}\text { Zagrabska- } \\
\text { Swiatkowska, et } \\
\text { al., (2020) }\end{array}$ & $\begin{array}{l}\text { (PPVT) e } \\
\text { Linha de } \\
\text { base }\end{array}$ & Mais e Menos & Imagens & Sim & - & - & - & 3 & Mesa & Sim \\
\hline
\end{tabular}

duras relacionais de hierarquia com transformação de funções. A tese consistiu em cinco diferentes capítulos, alguns apresentando discussões teóricas ou revisões de literatura e outros apresentando experimentos. Somente dois dos experimentos descritos foram publicados. No capítulo quatro, foram apresentados três experimentos, tendo o primeiro deles sido publicado em 2012, no International Journal of Psychology and Psychological Therapy. Os demais experimentos (Experimentos 2 e 3), apresentaram replicações modificadas do Experimento 1, sempre buscando resolver limitações observadas. No capítulo cinco, é apresentado o Experimento 4, uma replicação modificada do Experimento 3 que foi publicado em 2014 no International Journal of Psychology and Psychological Therapy. O Capítulo seis, apresenta os dados de dez pilotos realizados, para o estabelecimento dos procedimentos dos experimentos descritos nos capítulos anteriores. $\mathrm{O}$ trabalho realizado por Gil (2011) apresentou uma maneira inovadora de trabalhar com dicas contextuais de hierarquia, especialmente as dicas relacio- nais de "inclui" e "pertence a", em combinação com diversas outras dicas contextuais já amplamente investigadas (e.g., coordenação, diferença, comparação). Apesar de se tratar de um material muito rico, que permite um contato com o processo de desenvolvimento dos procedimentos realizados, iremos nos aprofundar nos procedimentos apenas dos dois experimentos publicados (i.e., Experimentos 1 e 4).

Gil, Luciano, Ruiz e Valdivia-Salas (2012) tiveram como objetivo analisar a transformação de funções que ocorrem entre os membros de três diferentes classes hierarquicamente organizadas. Este procedimento foi realizado com 10 participantes adultos e as tentativas foram apresentadas em um computador. Por meio do TME, no qual quatro estímulos foram treinados conforme as dicas contextuais de incluem, pertence a, mesmo e diferente. Posteriormente, duas categorias hierárquicas de 3 níveis foram treinadas por meio das dicas contextuais previamente treinadas e assim uma rede relacional de hierarquia foi então derivada e a transformação de função foi avaliada para 
os elementos dentro da rede relacional estabelecida. As funções transformadas nas redes relacionais hierárquicas estabelecidas foram "frio", "pesado" e "doce". Estas funções foram estabelecidas estrategicamente para permitir uma melhor avaliação da transformação de funções. Em uma rede hierárquica com duas subdivisões $(\mathrm{X})$ as funções foram atribuídas a um elemento hierárquico médio na primeira subdivisão (nesse caso esperava-se a transformação para o nível mais baixo) e na segunda foi já atribuído a um elemento no nível mais baixo (em que se esperava o compartilhamento dessa função nesse nível, mas não para os níveis superiores). Em uma outra rede hierárquica de três níveis, essas propriedades foram também atribuídas como no ultimo caso, no nível mais baixo e era prevista a transformação entre esses membros, mas não para os elementos hierarquicamente superiores. Os resultados permitiram observar a transformação de funções prevista no responder relacional derivado de oito dos dez participantes, cinco deles já na primeira apresentação do teste, e os outros três participantes, na segunda apresentação do teste.

Em um experimento posterior, Gil, Luciano, Ruíz e Valdivia-Salas. (2014) replicaram o estudo de 2012, com um procedimento bastante semelhante. As modificações implementadas nesse estudo foi uma ampliação das possibilidades de controle contextual. No experimento anterior, a instrução apresentada no estabelecimento de funções psicológicas que seria testada a transformação de função posteriormente foi " $\mathrm{X}$ é frio e pesado", o que tornava muito restrita a transformação de algumas funções. Neste experimento de 2014 a instrução utilizada foi "X tem uma parte que é fria e uma parte que é pesada". Além disso, as relações de níveis hierárquicos superiores, no estudo anterior haviam sido treinadas nas duas direções o que não permitiu a avaliação precisa de sua derivação. Nesse estudo elas foram treinadas em um sentido e testado no outro, para poder avaliar isoladamente sua derivação. As demais características do procedimento se mantiveram idênticas. Participaram desse experimento seis adultos. Foi possível observar nos seus resultados a transformação de funções esperada, para três deles no primeiro teste e para outros dois na segunda apresentação do teste.
Posteriormente aos estudos de Gil (2011), Slattery e Stewart (2014) se propuseram a ensinar a categorização hierárquica controlada contextualmente, a partir do treino de múltiplos exemplares envolvendo, primeiramente, treino de relações não-arbitrárias entre estímulos. Os estudos de Slattery e Stewart (2014) buscaram modelar propriedades de categorização hierárquica de acordo com o desenvolvimento cognitivo, a saber, pertencimento de classe transitiva, pertencimento de classe assimétrica (isto é, um classe de nível superior contém uma classe de nível hierárquico inferior, mas a classe inferior não contém a superior), e indução unilateral de propriedades (ou seja, as propriedades de uma classe de nível superior devem ser encontradas em uma classe de nível inferior, mas propriedades atribuídas a elementos de níveis hierarquicamente inferiores não devem ser observados nas classes de nível superior).

Esse trabalho contou com dois experimentos diferentes, ambos realizados no computador. O primeiro, em que quatro participantes adultos foram expostos a uma tarefa que incluía estímulos de múltiplas dimensões, com o objetivo de demonstrar o responder relacional hierárquico em três níveis diferentes. O segundo experimento contou com seis participantes adultos que realizaram todas as tarefas experimentais. A diferença deste estudo é que ele continha um conjunto de estímulos difuso ("fuzzy") para avaliar de forma mais controlada a transformação de funções. Os dois experimentos demonstraram com sucesso (todos os quatro participantes do Experimento 1 e cinco dos seis do Experimento 2) o estabelecimento de relações hierárquicas e das três diferentes propriedades de categorização hierárquica investigadas (pertencimento de classe transitiva e assimétrica, e também a indução unilateral de propriedades). Assim esses dois estudos demonstram que o emoldurar relacional hierárquico pode configurar um bom modelo para explicação da categorização hierárquica. Posteriormente, Stewart et al. (2018) utilizou o mesmo procedimento com estímulos com múltiplas dimensões para treinar relações hierárquicas com as dicas contextuais "parte-todo" e "inclui". Dez participantes foram expostos a esse procedimento demonstrando as propriedades definidoras das molduras relacionais 
hierárquicas, contudo o resultado da transformação de funções previsto não foi observado.

Dos estudos citados até aqui, todos os participantes foram adultos com desenvolvimento típico. $\mathrm{O}$ primeiro estudo que investigou esse tipo de responder relacional com crianças foi Mulhern, Stewart e McElwee (2017). 50 crianças de cinco faixas etárias diferentes (3-4 anos, 4-5 anos, 5-6 anos, 6-7 anos e 7-8 anos) foram testadas em procedimentos que avaliariam as propriedades definidoras de molduras relacionais, i.e., implicação mútua, implicação mútua combinatória e transformação de funções, em respostas a elementos de redes relacionais hierárquicas arbitrárias e não arbitrárias. Essas avaliações foram realizadas com estímulos (formas geométricas) impressos. Além disso, estas crianças foram expostas a três testes padronizados (PPVT4, SB5 e CCT). Os resultados demonstraram forte correlação entre os testes relacionais hierárquicos e os testes padronizados. Considerando que nenhum treino específico foi realizado, esse experimento não foi considerado na presente análise.

Posteriormente, o mesmo grupo de autores publicou o primeiro artigo que apresentou treinos relacionais de hierarquia com crianças com desenvolvimento típico. Mulhern, Stewart e McElwee (2018) realizaram dois experimentos com crianças com desenvolvimento típico, sendo no primeiro experimento, seis crianças de cinco a seis anos, e no segundo experimento, seis crianças de seis a sete anos. No primeiro experimento, foi realizada uma avaliação de responder relacional não arbitrário de pertencimento incluindo como estímulos caixas 3D de cores variadas. As avaliações do responder relacional arbitrário de pertencimento eram constituídas por sílabas sem sentido apresentados em um computador. Nas fases de treino e teste, os participantes deveriam responder com "sim" ou "não" para avaliar a resposta derivada bidirecional. A avaliação relacional inicial indicou que os seis participantes atingiram critério (acima de $80 \%$ de respostas corretas) para a avaliação de pertencimento não-arbitrária. Ao mesmo tempo, os seis falharam na avaliação de pertencimento arbitrária (nenhum pontuando acima de 60\%). Posteriormente, três dos participantes realizaram o treino relacional hierárquico e obtiveram critério de acerto nas fases treinadas. Os participantes que realizaram o treino e os que não realizaram foram testados para repertório relacional de pertencimento arbitrário após seis meses da interrupção do treino. Os resultados deste follow-up indicaram que os três participantes de treino mantiveram altos níveis de responder relacional de pertencimento, enquanto os participantes do grupo controle mostraram ganhos mínimos em desempenho quando comparados a avaliação inicial (pré-teste). O segundo experimento foi uma replicação do anterior. A diferença foi apenas uma faixa etária ligeiramente superior neste segundo. Os resultados foram similares aos observados anteriormente em relação ao sucesso no estabelecimento das relações hierárquicas e os ganhos mais robustos no follow-up, observado nos dados do grupo que foi exposto aos treinos.

O estudo de Ming et al. (2018) teve como participante de pesquisa três crianças com desenvolvimento típico e três crianças com desenvolvimento atípico, com idades equiparadas a partir da avaliação PPVT-4. Teve-se como objetivo treinar inclusão de classe. O procedimento foi realizado utilizando cartões impressos de quatro categorias diferentes (animais, roupas, frutas e veículos) e potes de plástico. O procedimento foi dividido em duas fases de treinos de múltiplos exemplares (a primeira, com suportes não-arbitrários e a segunda, sem a utilização destes) e uma fase de avaliação. A proposta foi utilizar inicialmente estímulos para verificar o pertencimento da classe não arbitrário que guiou a aquisição de um repertorio com elementos abstratos. A inclusão de classe foi avaliada em tentativas em que os participantes eram perguntados, por exemplo, se "existem mais cavalos ou animais?" Os resultados demonstraram que todos os participantes adquiriram as respostas de inclusão de classe e demonstraram generalização e manutenção após períodos de 4 a 6 semanas.

Posteriormente, Zagrabska-Swiatkowska et al. (2020) estende os estudos de Ming et al. (2018) realizando o ensino de respostas de inclusão de classe, tendo como participantes, três participantes adultos com diagnóstico de TEA. Os procedimentos foram os mesmos que os realizados anteriormente com os cartões impressos e potes plásticos sendo empregados para realização de um treino de múltiplos exemplares de três fases. As principais modificações realizadas nesse estudo foi verificar a impor- 
tância isolada do feedback individual contingente a resposta e a possibilidade da aplicação sem o uso dos suportes não-arbitrários. Os resultados apresentaram que, inicialmente, os participantes não demonstraram a aprendizagem com o uso de feedback individual contingente a resposta somente. A utilização do suporte não-arbitrário foi imprescindível para facilitar a aprendizagem das respostas de inclusão de classes.

\section{Discussão}

A partir desta revisão fica claro o desenvolvimento da proposta, no escopo da Teoria das Molduras Relacionais (Hayes, el. al., 2001), para apresentar a definição funcional da categorização hierárquica. Foi possível observar, a partir de diversas evidências (Slattery et al., 2011, Gil et al., 2012; 2014) como as propriedades definidoras do RRAA são observadas nesse tipo de moldura relacional, especialmente a transformação de função e a assimetria esperada em diferentes níveis hierárquicos. Além disso, esses trabalhos também procuraram investigar variáveis características deste tipo de repertório, consideradas importantes a partir de outras bases teóricas, como o pertencimento de classe transitiva, pertencimento de classe assimétrica e indução unilateral de propriedades (Slattery \& Stewart, 2014) ou mesmo a inclusão de classes (Ming et al., 2018; Zagrabska-Swiatkowska et al., 2020). Os resultados observados ressaltaram o potencial e a precisão da explicação baseada em molduras relacionais de hierarquia.

O conjunto de trabalhos aqui descritos, permitiu também apresentar algumas caraterísticas importantes deste repertório. Em relação a média de idade em que este tipo de relação é estabelecido observamos algumas evidências que merecem destaque. No contexto da Análise do Comportamento, de acordo com o VB-MAPP (Sundberg, 2008), a partir dos 2 anos e meio as crianças já são capazes de apresentar domínios para responder sob classe, função e características. Podemos inferir que repostas nesse marco específico poderiam já corresponder a categorização hierárquica, contudo no trabalho de Mulhern et al. (2017), nenhuma das dez crianças com faixa etária de 3-4 anos demonstrou nenhum tipo de resposta relacional derivada na moldura de hierarquia. Nesse ponto, fica claro que a definição baseada em responder relacional derivado nos permite uma diferenciação funcional precisa. Possivelmente crianças a partir dos 2 anos e meio sejam capazes de dizer que "gatos são felinos". Por outro lado, a dica contextual relacional nesse exemplo poderia ser tanto a de coordenação como hierarquia. Apesar de destacar a idade, não estamos sugerindo que variáveis maturacionais sejam essenciais, mas apontamos como o responder baseado em molduras de hierarquia apresenta desafios de outro nível.

Além disso, é reconhecido na literatura da área que esse tipo de repertório depende de molduras relacionais de menor complexidade relacional, muito provavelmente por essa razão ele tem sido observado mais tardiamente no desenvolvimento infantil típico. Trabalhos futuros como esse de Mulhern e colaboradores, com o objetivo de mapear esse repertório olhando para seus aspectos funcionais, podem contribuir grandemente com intervenções em categorização hierárquica. Procedimentos de análise funcional de repertórios comportamentais permitem que o ensino seja mais adequado ao repertório de entrada do indivíduo que está no papel de aprendiz, e o procedimento ganha em eficiência, por estar direcionado para as lacunas de um repertório comportamental individual.

A categorização hierárquica representa um repertório comportamental relevante e ainda pouco explorado em estudos analítico-comportamentais. Esse baixo índice de investigação neste tema pode ser comprovado pela baixa quantidade de estudos encontrados nessa revisão. A partir da observação da quantidade de trabalhos interessados nesse repertório em indivíduos com algum tipo de atraso no desenvolvimento, a quantidade de estudos é ainda mais restrita. É clara a relevância das investigações com população com desenvolvimento típico favorecerem esclarecimentos sobre como se dá o estabelecimento e manutenção dessas relações no desenvolvimento infantil típico, e da contribuição das mesmas para a expansão do saber científico. No entanto, essas investigações não são suficientes para indicar procedimentos efetivos de ensino para a população com autismo. Somente quatro estudos dos quinze encontrados investigaram relações hierárquicas nessa população, o que indica a urgência em novas investigações desta natureza. 
Os procedimentos realizados por Mulhern et al., (2017; 2018), Ming et al., 2018; ZagrabskaSwiatkowska et al. (2020) incluíram sempre como participantes crianças, e em alguns destes estudos crianças com atrasos no desenvolvimento. Além disso, trabalhos como o de Gil et al. $(2012 ; 2014)$ e Slattery e Stewart (2014) possuem procedimentos relativamente pontuais que poderiam ser facilmente adaptáveis para crianças com desenvolvimento típico ou atípico. A racional destes dois últimos estudos sempre envolveu ensinar elementos abstratos como dicas contextuais relacionais hierárquicas para estabelecer relações arbitrárias de hierarquia. Os treinos de múltiplos exemplares empregados, possivelmente poderiam ser adaptados para crianças mais velhas e talvez o uso de dicas contextuais de hierarquia culturalmente estabelecidas como por exemplo "está contido", “contém", “é parte de", entre outras favoreceria ainda mais o desenvolvimento deste repertório.

Em relação às características de procedimento, Mulhern et al., (2018) empregou como dicas não-arbitrárias o uso de potes plásticos transparentes e o uso de itens tridimensionais familiares aos participantes. Esse é outro recurso que decisivamente amplia a possibilidade de aplicação com crianças mais novas ou com algum atraso de desenvolvimento. No estudo em que esse tipo de procedimento foi desenvolvido o foco foi exclusivamente para relações do tipo não arbitrárias entre estímulos. Estudos futuros poderiam investigar, não somente como esse aprendizado ocorre, mas o quanto esses elementos familiares e relações não-arbitrárias poderiam funcionar como um treino prévio capacitando um responder relacional de maior complexidade entre elementos arbitrários posteriormente.

Considerando a expectativa de fomentar estudos na área com a população com autismo, Zagrabska-Swiatkowska et al. (2020) indicam que pesquisas futuras possam explorar em que medida a inclusão de classe pode ser treinada e generalizada em indivíduos com diagnóstico de atraso no desenvolvimento que apresentam níveis mais baixos de desenvolvimento linguístico do que os observados no estudo. Brooks, Newsome et al. (2014) sugerem a possibilidade de ampliação das tentativas de exposição ao treino de múltiplos exemplares aos participantes com autismo. Estes estudos preliminares da população com autismo denotam como a RFT fornece uma base teórica útil que pode contribuir para produção de tecnologias eficazes, que possam colaborar para o ensino de repertórios verbais complexos. A forma integrativa com que a RFT descreve o repertório verbal e como a unidade de análise do RRAA é relevante para diferentes comportamentos, permite inferir que o desenvolvimento em molduras hierárquicas permitirá a evolução em diversos outros tipos de controle contextual. Adicionalmente, indicar intervenções efetivas a essa população tem como expectativa garantir ainda mais a validade social dos estudos que estão sendo produzidos na área.

Por fim, fica claro por essa revisão que ainda existe muito a ser investigado em relação a complexidade da resposta relacional hierárquica. Os resultados das pesquisas aqui apresentados contribuem para a compreensão dos repertórios relacionais derivados e indicam serem promissores para contribuir para o desenvolvimento de um currículo de ensino para intervenção em categorização hierárquica e, consequentemente, para linguagem de crianças e adultos com autismo e outros atrasos de desenvolvimento. Fortalecer o repertorio relacional hierárquico de indivíduos com TEA pode impactar em inúmeros ganhos secundários, como sua autonomia, na organização de seus itens pessoais e de categorização de indivíduos em suas relações interpessoais, como também tem uma grande ligação com inúmeras habilidades intelectuais e acadêmicas, bem como na compreensão de interações verbais complexas (Gale \& Stewart, 2020; Lovaas, 1987).

\section{Referências}

American Psychiatric Association. (2014). DSM-5: Manual diagnóstico e estatístico de transtornos mentais. Artmed Editora.

Brooks Newsome, K., Nix Berens, K., Ghezzi, P. M., Aninao, T., \& Newsome, W. D. (2014). Training relational language to improve reading comprehension. European Journal of Behavior Analysis, 15(2), 165-197. https://doi.org/10.108 $0 / 15021149.2014 .11434512$

Carneiro, P., Albuquerque, P., \& Fernandez, A. (2009). Opposite developmental trends for 
false recognition of basic and superordinate names. Memory, 17, 411-427. https://doi. org/10.1080/09658210902758847

Eimas, P. D., \& Quinn, P. C. (1994). Studies on the formation of perceptually based basic-level categories in young infants. Child Development, 65(3), 903-917. https://doi. org/10.2307/1131427

Foody, M., Barnes-Holmes, Y., Barnes-Holmes, D., Rai, L., \& Luciano, C. (2015). An empirical investigation of the role of self, hierarchy, and distinction in a common act exercise. The Psychological Record, 65(2), 231-243. https:// doi.org/10.1007/s40732-014-0103-2

Gale, L., \& Stewart, I. (2020). Assessing and Training Comparative Relations in Children with Autism Spectrum Disorder. Journal of European Psychology Students, 11(1), 1-14. https://doi.org/10.5334/jeps.487

Gil, E., Ruiz, F. J., Luciano, C., \& Valdivia-Salas, S. (2012). A preliminary demonstration of transformation of functions through hierarchical relations. International Journal of Psychology and Psychological Therapy, 12 (1), 1-19

Gil-Luciano, B., Ruiz, F. J., Valdivia-Salas, S., \& Suárez-Falcón, J. C. (2017). Promoting psychological flexibility on tolerance tasks: Framing behavior through deictic/hierarchical relations and specifying augmental functions. The Psychological Record, 67(1), 1-9. https://doi. org/10.1007/s40732-016-0200-5.

Gil, E. (2011). Transformación de funciones a través del marco relacional de jerarquía. Tese de Doutorado não publicada. Universidad de Almería.

Gil, E., Ruiz, F. J., Luciano, C., \& Valdivia-Salas, S. (2014). A further experimental step in the analysis of hierarchical responding. International Journal of Psychology and Psychological Therapy, 14 (2), 137-153.

Hayes, S. C. Barnes-Holmes, D., \& Roche, B. (2001). Relational Frame Theory: A Post-Skinnerian account of human language and cognition. New York: Plenum Press.

Laipelt, R., C., F., \& Krebs, L., M., (2018) Teorias da linguística cognitiva para pensar a categorização no âmbito da ciência da informação. Transinformação,30, 81-93. https://doi. org/10.1590/2318-08892018000100007.

López-López, J. C., \& Luciano, C. (2017). An experimental analysis of defusion interactions based on deictic and hierarchical framings and their impact on cognitive performance. The Psychological Record, 67(4), 485-497. http://doi. org/10.1007/s40732-017-0250-3

Lovaas, O. I. (1987). Behavioral treatment and normal educational and intellectual functioning in young autistic children. Journal of consulting and clinical psychology, 55(1), 3. http://doi. org/10.1037//0022-006x.55.1.3

McCabe, A. E., Siegel, L. S., Spence, I., \& Wilkinson, A. (1982). Class-inclusion reasoning: Patterns of performance from three to eight years. Child Development, 780-785.

McHugh, L., \& Reed, P. (2008). Using Relational Frame Theory to build grammar in children with Autistic Spectrum Conditions. The Journal of Speech and Language PathologyApplied Behavior Analysis, 3(1), 60. http://doi. org/10.1037/h0100233

Ming, S., Mulhern, T., Stewart, I., Moran, L., \& Bynum, K. (2018). Training class inclusion responding in typically developing children and individuals with autism. Journal of applied behavior analysis, 51(1), 53-60. http://doi. org/10.1002/jaba.429

Mulhern, T., Stewart, I., \& McElwee, J. (2017). Investigating relational framing of categorization in young children. The Psychological Record, 67(4), 519-536. http://doi.org/10.1007/ s40732-017-0255-y

Mulhern, T., Stewart, I., \& McElwee, J. (2018). Facilitating relational framing of classification in young children. Journal of contextual behavioral science, 8, 55-68. https://doi. org/10.1016/j.jcbs.2018.04.001

Murphy, G. L., (2002) The big book of concepts. MIT Press.

Perez, W. F., Nico, Y. C., Kovac, R., Fidalgo, A. P., \& Leonardi, J. L. (2013). Introdução à Teoria das Molduras Relacionais (Relational Frame Theory): principais conceitos, achados experimentais e possibilidades de aplicação. Perspectivas em análise do comportamento, 4(1), 32-50. https://doi.org/10.18761/ perspectivas.v4i1.105 
Persicke, A., Tarbox, J., Ranick, J., \& Clair, M. S. (2012). Establishing metaphorical reasoning in children with autism. Research in Autism Spectrum Disorders, 6(2), 913-920. https://doi. org/10.1016/j.rasd.2011.12.007

Slattery B., Stewart I., O’Hora D. (2011) Testing for transitive class containment as a feature of hierarchical classification. Journal of the Experimental and Analysis of Behavior, 96, 243-260. http://doi. org/10.1901/jeab.2011.96-243

Slattery, B., \& Stewart, I. (2014). Hierarchical classification as relational framing. Journal of the Experimental Analysis of Behavior, 101(1), 6175. http://doi.org/10.1002/jeab.63

Stewart, I., Slattery, B., Chambers, M., \& Dymond, S. (2018). An empirical investigation of part-whole hierarchical relations. European Journal of Behavior Analysis, 19(1), 105-124. http://doi. org/10.1080/15021149.2017.1416525

Zagrabska-Swiatkowska, P., Mulhern, T., Ming, S., Stewart, I., \& McElwee, J. (2020). Training class inclusion responding in individuals with autism: Further investigation. Journal of Applied Behavior Analysis, 53(4), 2067-2080. https:// doi.org/10.1002/jaba.712 